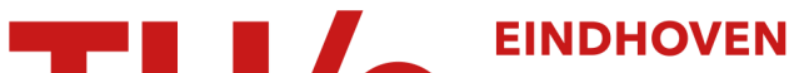 UNIVERSITY OF TECHNOLOGY
}

\section{Mode-dependent Loss and Gain Emulation in Coupled SDM Transmission}

Citation for published version (APA):

Ospina, R. S. B., van den Hout, M., Okonkwo, C. M., \& Mello, D. A. A. (2020). Mode-dependent Loss and Gain Emulation in Coupled SDM Transmission. Paper presented at XXXVIII Simpósio Brasileiro de

Telecomunicações e Processamento de Sinais (SBrT 2020). https://doi.org/10.14209/SBRT.2020.1570658187

DOI:

10.14209/SBRT.2020.1570658187

Document status and date:

Published: 22/11/2020

Document Version:

Publisher's PDF, also known as Version of Record (includes final page, issue and volume numbers)

Please check the document version of this publication:

- A submitted manuscript is the version of the article upon submission and before peer-review. There can be important differences between the submitted version and the official published version of record. People interested in the research are advised to contact the author for the final version of the publication, or visit the $\mathrm{DOI}$ to the publisher's website.

- The final author version and the galley proof are versions of the publication after peer review.

- The final published version features the final layout of the paper including the volume, issue and page numbers.

Link to publication

\section{General rights}

Copyright and moral rights for the publications made accessible in the public portal are retained by the authors and/or other copyright owners and it is a condition of accessing publications that users recognise and abide by the legal requirements associated with these rights.

- Users may download and print one copy of any publication from the public portal for the purpose of private study or research.

- You may not further distribute the material or use it for any profit-making activity or commercial gain

- You may freely distribute the URL identifying the publication in the public portal.

If the publication is distributed under the terms of Article $25 \mathrm{fa}$ of the Dutch Copyright Act, indicated by the "Taverne" license above, please follow below link for the End User Agreement:

www.tue.nl/taverne

Take down policy

If you believe that this document breaches copyright please contact us at:

openaccess@tue.nl

providing details and we will investigate your claim. 


\title{
Mode-dependent Loss and Gain Emulation in Coupled SDM Transmission
}

\author{
Ruby S. B. Ospina, Menno van den Hout, Chigo Okonkwo and Darli A. A. Mello
}

\begin{abstract}
Space-division multiplexing (SDM) is currently the only solution to cope with the exponential growth of data traffic in optical transmission networks. The performance of long-haul SDM transmission is fundamentally limited by mode-dependent loss (MDL) and mode-dependent gain (MDG) generated in components and amplifiers. To enable the study of MDL/MDG effects in SDM systems as well as MDL/MDG estimation methods within the context of experimental setups, we evaluate an MDL/MDG emulator based on variable optical attenuators (VOAs) and photonic lanterns. We assess MDL/MDG emulation in different attenuation scenarios and demonstrate the capability of the emulator to artificially introduce a wide range of MDL/MDG in a short-reach 3-mode transmission system.
\end{abstract}

Keywords-Mode-dependent loss, mode-dependent gain, space-division multiplexing, long-haul transmission.

\section{INTRODUCTION}

Space-division multiplexing (SDM) has been regarded as the only solution to sustain future requirements of information traffic in optical core networks [1], [2]. Optical SDM can be implemented over bundles of single-mode fibers (SMFs) [3], [4], multi-core fibers (MCFs) [5], multi-mode fibers (MMFs) [6] or few-mode multi-core fibers [7]. Coupled SDM transmission over multi-mode fibers or coupled-core multi-core fibers (CC-MCFs) [8], [9] demands adaptive multiple-input multipleoutput (MIMO) equalizers at reception to compensate for linear coupling among the propagating modes. Unlike mode coupling, mode-dependent loss (MDL) and mode-dependent gain (MDG) generated in components and amplifiers are not compensated by digital signal processing (DSP). MDL/MDGinduced random power variations of the guided modes turn the channel capacity into a random variable, reducing the average capacity and generating outages. Accumulated MDL/MDG poses fundamental performance limitations to high-capacity SDM systems deployed at long distances. In [10], Ho et al. characterize statistically the MDL effect and analyze its impact on the channel capacity in strongly coupled SDM systems. In [11], Winzer et al. also discuss the MDL-induced capacity reduction in SDM channels and study the system outage performance. In [12], Mello et al. study the effect of frequency diversity on the MDG-induced outage probability and quantify the maximum tolerable per-amplifier MDG for a certain average capacity metric in ultra-long-haul systems. MDL/MDG management is usually addressed at component design and fabrication levels. In [13], [14], Lobato et al. review alternative approaches to relieve MDL/MDG effects.

As the achievable channel capacity and transmission distance in SDM systems are fundamentally constrained by MDL/MDG, the study of the MDL/MDG impact on the system performance as well as MDL/MDG estimation methods should be performed in laboratory complex setups. Alternatively, as multiple multi-mode amplifier spans or recirculating loops may not be available, accumulated MDL/MDG can be artificially introduced in a smaller-scale experimental setup. In [15], Mizuno et al. emulate MDL using attenuators and mode multiplexers based on planar lightwave circuits (PLCs). However, mode multiplexers based on photonic lanterns (PLs) are currently regarded as the most promising solution for mode multiplexing. Therefore, MDL/MDG emulation must be aligned with the potential integration of these devices in practical SDM systems.

In this work, we experimentally evaluate an MDL/MDG emulator based on variable optical attenuators (VOAs) and modemultiplexers and demultiplexers based on photonic lanterns. The MDL/MDG emulator is implemented to introduce accumulated MDL/MDG in a short-reach 3-mode (32.5 meters) fiber link. We assess MDL/MDG emulation at different VOA configurations and demonstrate the capacity of the emulator to provide a wide range of MDL/MDG as a function of the difference among mode launch powers.

\section{MDL/MDG FUNDAMENTALS}

High capacity SDM optical systems deployed at long distances require multiple inline amplifiers that may have a nonflat gain profile over guided spatial modes, resulting in MDG. Moreover, fiber bends, connectors, splices, and optical devices, such as multiplexers, introduce MDL. Usually, analytical models for SDM systems are employed to study the MDL/MDG impact in numerical and simulation contexts [10], [16]. The multi-section model proposed in [10] splits the fiber link into $\mathrm{K}$ sections. At an angular frequency $\omega$, the transfer matrix of the $k^{\text {th }}$ section, $\mathbf{H}^{(\mathrm{k})}(\omega)$, can be modeled as the product of $3 \mathrm{D} \times \mathrm{D}$ matrices, with $\mathrm{D}$ being the number of orthogonal modes (spatial and polarization modes)

$$
\mathbf{H}^{(\mathrm{k})}(\omega)=\mathbf{V}^{(\mathrm{k})} \boldsymbol{\Lambda}^{(\mathrm{k})}(\omega) \mathbf{U}^{(\mathrm{k}) H}, \quad \text { with } \mathrm{k}=1, \ldots, \mathrm{K},
$$

where $(.)^{H}$ denotes the Hermitian transpose operator, and $\mathbf{V}$ and $\mathbf{U}$ are frequency-independent random unitary matrices that describe mode coupling at the input and output of the span, respectively. The diagonal matrix $\Lambda^{(\mathrm{k})}(\omega)$ that accounts for MDL/MDG is given by

$\boldsymbol{\Lambda}^{(\mathrm{k})}(\omega)=\operatorname{diag}\left[\mathrm{e}^{\frac{1}{2} \mathrm{~g}_{1}^{(\mathrm{k})}-\mathrm{j} \omega \tau_{1}^{(\mathrm{k})}}, \mathrm{e}^{\frac{1}{2} \mathrm{~g}_{2}^{(\mathrm{k})}-\mathrm{j} \omega \tau_{2}^{(\mathrm{k})}}, \ldots, \mathrm{e}^{\frac{1}{2} \mathrm{~g}_{\mathrm{D}}^{(\mathrm{k})}-\mathrm{j} \omega \tau_{\mathrm{D}}^{(\mathrm{k})}}\right]$,

where

$$
\mathrm{g}^{(\mathrm{k})}=\left(\mathrm{g}_{1}^{(\mathrm{k})}, \mathrm{g}_{2}^{(\mathrm{k})}, \ldots, \mathrm{g}_{\mathrm{D}}^{(\mathrm{k})}\right)
$$


and

$$
\tau^{(\mathrm{k})}=\left(\tau_{1}^{(\mathrm{k})}, \tau_{2}^{(\mathrm{k})}, \ldots, \tau_{\mathrm{D}}^{(\mathrm{k})}\right),
$$

are the uncoupled log-power gains and the uncoupled modal groups delays, respectively. The overall transfer matrix $\mathbf{H}(\omega)$ of a fiber of $\mathrm{K}$ spans is given by

$$
\mathbf{H}(\omega)=\prod_{\mathrm{k}=1}^{\mathrm{K}} \mathbf{H}^{(\mathrm{k})}(\omega)
$$

The transfer matrix $\mathbf{H}(\omega)$ can be also expressed as the product of three matrices as

$$
\mathbf{H}(\omega)=\mathbf{V}(\omega) \boldsymbol{\Lambda}(\omega) \mathbf{U}^{H}(\omega)
$$

where $\mathbf{V}$ and $\mathbf{U}$ are unitary matrices, and $\boldsymbol{\Lambda}$ is a diagonal matrix. The overall coupled modal gains $\mathbf{g}=\left(\mathrm{g}_{1}, \mathrm{~g}_{2}, \ldots, \mathrm{g}_{\mathrm{D}}\right)$ contained at the main diagonal of $\boldsymbol{\Lambda}(\omega)$ characterize the system MDL/MDG. The overall coupled modal gains in logpower-gain units are given by the logarithm of the eigenvalues $\lambda_{i}^{2}$ of the operator $\mathbf{H}(\omega) \mathbf{H}^{H}(\omega)$. In the strong coupling regime, the overall MDL/MDG is usually quantified by the standard deviation of the coupled modal, gains denoted by $\sigma_{\mathrm{mdg}}$ [10], [12], [17]. In links with weak mode coupling, the peak-to-peak MDL/MDG is a more relevant metric and is given by the ratio between the highest and the lowest eigenvalue $\lambda_{i}^{2}$ [11].

In a simulation context, the overall MDL/MDG is controlled through the MDG standard deviation per span, $\sigma_{\mathrm{g}}$. The accumulated MDL/MDG standard deviation, $\xi$, increases with the square-root of $\mathrm{K}$ as $\xi=\sigma_{\mathrm{g}} \sqrt{\mathrm{K}}$. The standard deviation of the overall MDL/MDG, and the accumulated MDL/MDG standard deviation, $\xi$, are related as

$$
\sigma_{\mathrm{mdg}}=\xi \sqrt{1+\frac{\xi^{2}}{12\left(1-\mathrm{D}^{-2}\right)}} .
$$

In SDM coherent transmission systems that employ MIMO equalizers to compensate for linear channel impairments, MDL/MDG is estimated from the inverse of the MIMO transfer matrix, $\mathbf{W}^{-1}$, as an estimate of $\mathbf{H}$ [18]. Therefore, the peak-to-peak MDL/MDG and $\sigma_{\mathrm{mdg}}$ are computed from the eigenvalues of the operator $\mathbf{W}^{-1}\left(\mathbf{W}^{-1}\right)^{H}$. Fig. 1 shows the peak-to-peak MDL/MDG and the overall $\sigma_{\mathrm{mdg}}$ in $\mathrm{dB}$ units computed from $\mathbf{H}$ and $\mathbf{W}^{-1}$ as a function of the number of spans in a 3-mode system with polarization multiplexing, $\sigma_{\mathrm{g}}=0.55 \mathrm{~dB}$, span length of $50 \mathrm{~km}$, group delay standard deviation $\sigma_{\tau}=3.1 \mathrm{ps} / \sqrt{\mathrm{km}}$ [19] and symbol rate of 25 Gbaud. Table I summarizes the main simulation parameters. In Fig. 1, $\sigma_{\text {mdg }}$ estimated from $\mathbf{H}$ and $\mathbf{W}^{-1}$ tracks the analytical curve given by (7) with negligible error.

\section{EXPERIMENTAL MDL/MDG EMULATOR}

In small-scale laboratory setups, long fiber links, recirculating loops and SDM amplifiers may not be available. In this case, MDL/MDG can be emulated by selective attenuation using VOAs preceding spatial multiplexing. Spatial mode multiplexers and demultiplexers play a critical role in SDM systems. Depending on the component type and design, these devices can have large insertion loss, strong

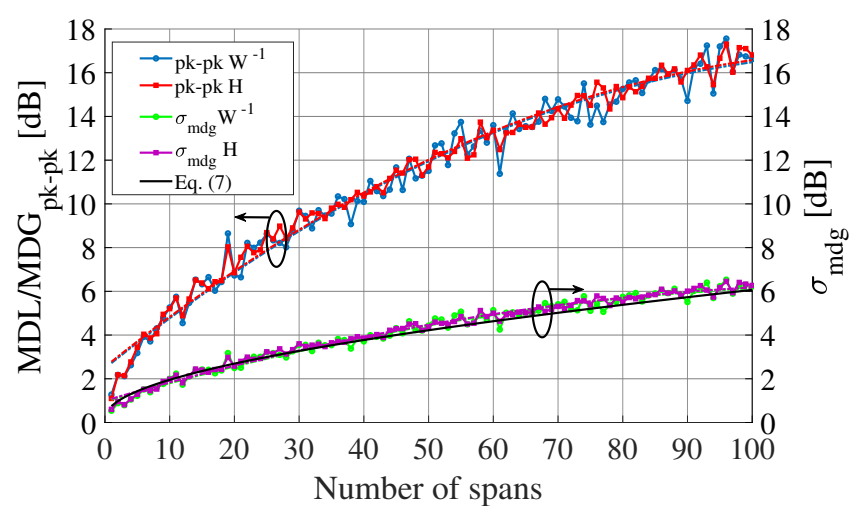

Fig. 1: Accumulated MDL/MDG as a function of the number of spans in a 3-mode system with polarization multiplexing. The MDL/MDG ${ }_{\mathrm{pk}-\mathrm{pk}}$ metric is shown at the left y-axis. The $\sigma_{\mathrm{mdg}}$ metric is shown at the right y-axis. Dashed lines show interpolated curves. In the legend, $\mathbf{H}$ is the channel transfer function and $\mathbf{W}$ is the equalizer filter coefficients.

TABLE I: Simulation parameters

\begin{tabular}{|l|c|}
\hline \multicolumn{1}{|c|}{ Parameter } & Value \\
\hline $\mathrm{D}$, number of orthogonal modes & 6 \\
\hline $\mathrm{K}$, number of spans & From 0 to 100 \\
\hline Span length & $50 \mathrm{~km}$ \\
\hline$\lambda_{i}^{2}$, eigenvalues of $\mathbf{H}(\omega) \mathbf{H}^{H}(\omega)$ & Variable \\
\hline$\sigma_{\mathrm{g}}$, MDG standard deviation per span & $0.55 \mathrm{~dB}$ \\
\hline$\xi$, MDG accumulated standard deviation & Variable \\
\hline$\sigma_{\mathrm{mdg}}$, standard deviation of the overall MDG & Variable \\
\hline$\sigma_{\tau}$, group delay standard deviation & $3.1 \mathrm{ps} / \sqrt{\mathrm{km}}$ \\
\hline Symbol rate & $25 \mathrm{GBaud}$ \\
\hline
\end{tabular}

power coupling, and high MDL. Photonic lanterns are low-loss passive optical waveguides that efficiently convert multi-mode light into multiple single-mode signals, or vice-versa [20]. The PL is a bundle of single-mode fibers adiabatically tapered to form a single glass body that progressively reduces its crosssectional dimension to form an MMF core. During the taper transition, light propagating in the single-mode cores evolves into a linear combination of the MMF modes at the lantern output [21]. Mode selectivity in PLs is achieved during the fabrication process, in which the use of different single-mode core diameters allows the selective excitation of a specific mode group [20]. Fig. 2 shows the transfer matrices of two 6-mode photonic lanterns in back-to-back configuration, with a section of 32.5 meter MMF between them, as shown in Fig. 2a. Fig. 2b corresponds to the transfer function in which the signals are transmitted in PL 2 and received in PL 1. In Fig. $2 \mathrm{c}$, the signals are transmitted in PL 1 and received in PL 2. The color bar indicates the power transmission from the TX port located in the vertical axis to the RX port located in the horizontal axis. The measurement process is performed by launching a signal with a known power at each port of the input PL and measuring the output power at all ports of the output PL in the configuration depicted in Fig. 2a. The power transmission is given by the ratio between the output power and the input power. From Figs. $2 b$ and $2 c$, both PLs have 


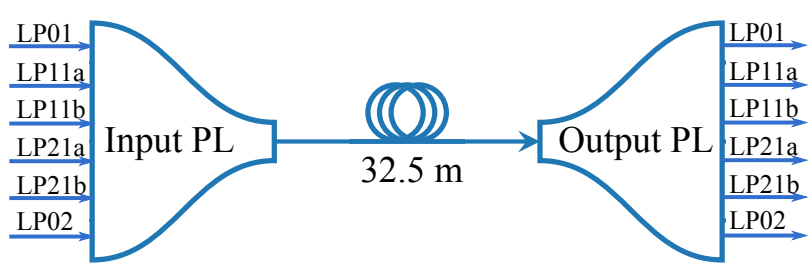

(a)

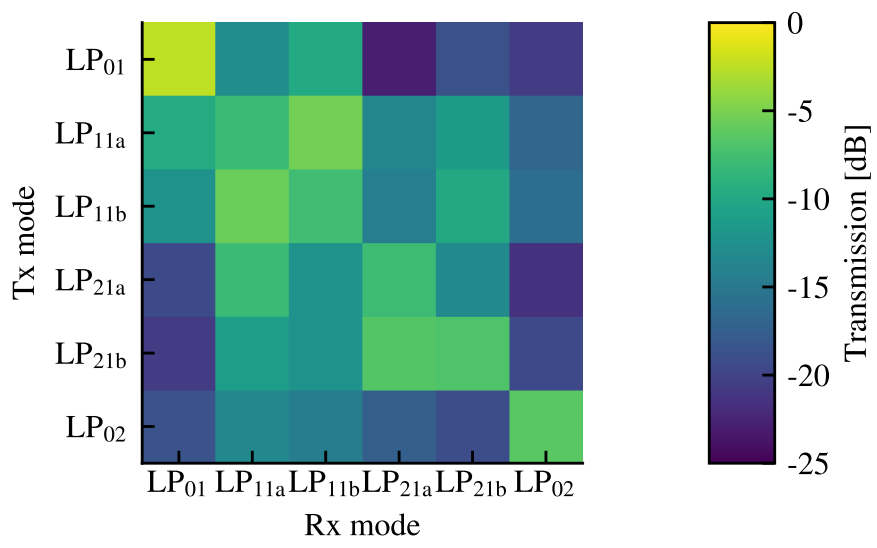

(b)
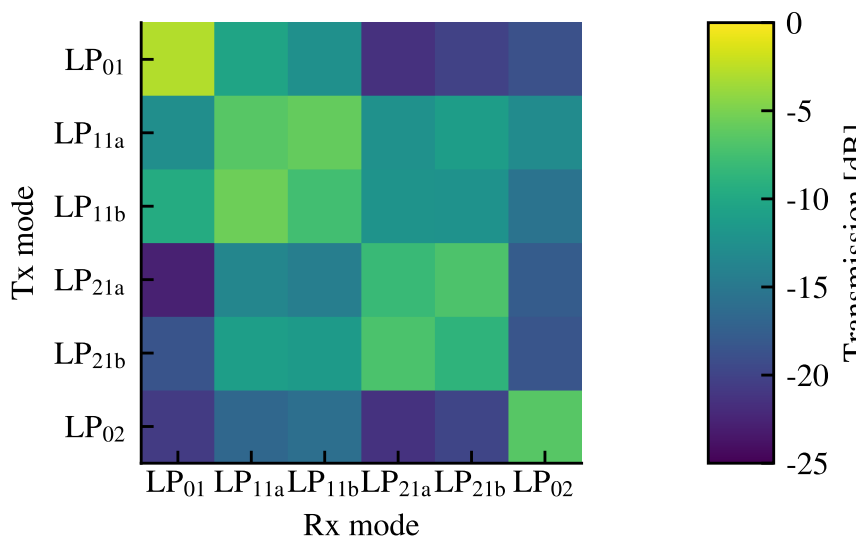

(c)

Fig. 2: Power transfer matrices of two photonic lanterns in back-to-back with a section of 32.5 meter MMF between them. (a) Experimental setup. (b) Transmission with PL 2 at the transmitter and PL 1 at the receiver. (c) Transmission with PL 1 at the transmitter and PL 2 at the receiver.

similar performance. For $\mathrm{LP}_{01}$ and $\mathrm{LP}_{02}$, most of the power launched at the input port is received at the corresponding port at the output PL, indicating low mode coupling. In the case of mode groups with degenerated modes "a" and " $b$ ", such as $\mathrm{LP}_{11}$ and $\mathrm{LP}_{21}$, although high levels of mode coupling exist between $\mathrm{LP}_{11 \mathrm{a}}$ and $\mathrm{LP}_{11 \mathrm{~b}}$, and $\mathrm{LP}_{21 \mathrm{a}}$ and $\mathrm{LP}_{21 \mathrm{~b}}$, the largest portion of energy remains within the same mode group as expected. In general, modes within the same mode group or with similar propagation constants experience strong mode coupling, while low mode coupling is experienced by modes whose propagation constants are highly unequal [22].

The experimental setup evaluated for MDL/MDG emulation based on VOAs and PLs is depicted in Fig. 3. At the transmitter, a pseudo-random bit sequence is generated and mapped to $2^{16} 16-\mathrm{QAM}$ symbols at $25 \mathrm{GBd}$. Pulse shaping at the transmitter is done using a root-raised-cosine (RRC) filter with 0.01 roll-off factor. The shaped signal is converted to analog by a 100-GSa/s digital-to-analog converter (DAC) followed by $40 \mathrm{GHz}$ (3dB bandwidth) RF-amplifiers. The analog signal modulates the output of an external cavity laser (ECL) with $80-\mathrm{kHz}$ bandwidth using a dual-polarization in-phase and quadrature modulator. After optical modulation, the signal is amplified by an erbium-doped fiber amplifier (EDFA), split and delayed to generate 3 decorrelated data streams that are passed through VOAs and then multiplexed by a mode-selective photonic lantern [20]. Although the used photonic lanterns and the fiber support six spatial modes $\left(\mathrm{LP}_{01}\right.$, $\left.\mathrm{LP}_{11 \mathrm{a}}, \mathrm{LP}_{11 \mathrm{~b}}, \mathrm{LP}_{21 \mathrm{a}}, \mathrm{LP}_{21 \mathrm{~b}}, \mathrm{LP}_{02}\right)$, only three are used for data transmission $\left(\mathrm{LP}_{01}, \mathrm{LP}_{11 \mathrm{a}}\right.$ and $\left.\mathrm{LP}_{11 \mathrm{~b}}\right)$. The VOAs allow the independent control of the launch powers in the 3 spatial modes $\mathrm{LP}_{01}, \mathrm{LP}_{11 \mathrm{a}}$ and $\mathrm{LP}_{11 \mathrm{~b}}$ to artificially introduce MDL/MDG into the system. The mode-multiplexed signal is coupled into a 50 $\mu \mathrm{m}$ core diameter graded-index few-mode fiber (GI-FMF) of 32.5 meters whose refractive index profile supports up to 6 linearly polarized (LP) modes at $1550 \mathrm{~nm}$. After propagation, the signals are demultiplexed by a second PL and sent to a time domain multiplexed (TDM)-SDM receiver [7] that delays two flows by $3 \mathrm{~km}$ and $6 \mathrm{~km}$ of standard single-mode fiber (SSMF) to reduce the complexity of the coherent receiver. After the TDM-SDM device, a noise loading stage composed of two EDFAs, a wavelength selective switch (WSS) and a VOA is placed to vary the OSNR at the coherent receiver input. The average OSNR is measured by an optical spectrum analyzer (OSA) after the last amplification stage. The noisy signal is amplified and converted from the optical to the electrical domain by the receiver front-end that integrates a second ECL as local oscillator (LO). The TDM electric signals are fed into 80-GSa/s analog-to-digital converters (ADC) to be digitized. In the DSP block, the TDM streams are parallelized and downsampled to two samples per symbol. Next, the frequency offset is estimated and compensated for. The signal is matchedfiltered by a RRC filter, and, finally, decision-directed (DD) equalization is applied. $6 \times 6$ MIMO equalization (processing 3 spatial modes, with 2 polarization modes each) is carried out using a widely linear complex-valued adaptive equalizer with 72 finite impulse response filters with 1001 taps each, updated by a fully supervised DD-LMS algorithm [23].

\section{MDL/MDG EMULATION RESULTS}

The experimental emulation of MDL/MDG is carried out by attenuating the single-mode signals at the input of the multiplexer PL. At $0 \mathrm{~dB}$ attenuation, the launch powers are 0.55 $\mathrm{dBm},-0.15 \mathrm{dBm}$ and $-0.15 \mathrm{dBm}$ for $\mathrm{LP}_{01}, \mathrm{LP}_{11 \mathrm{a}}$ and $\mathrm{LP}_{11 \mathrm{~b}}$, respectively. The system has an inherent $\mathrm{MDL} / \mathrm{MDG}_{\mathrm{pk}-\mathrm{pk}}=$ $3.9 \mathrm{~dB}$ and $\sigma_{\mathrm{mdg}}=1.3 \mathrm{~dB}$ coming from the different launch powers, imperfections of the optical splitters and different insertion losses of the VOAs. In order to keep the total launch power constant at $-4.9 \mathrm{dBm}$, the 3 VOAs are initialized in $5 \mathrm{~dB}$ attenuation to attenuate or de-attenuate the signals according to the configurations defined in Table II. In case 1, 


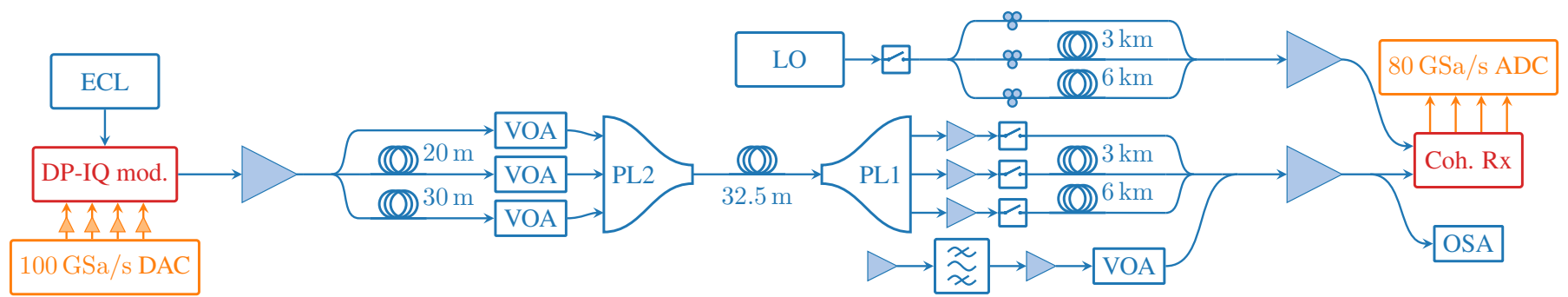

Fig. 3: Experimental setup for MDL/MDG emulation in a 3-mode transmission system with polarization multiplexing. The launch powers of the 3 modes $\left(\mathrm{LP}_{01}, \mathrm{LP}_{11 \mathrm{a}}\right.$ and $\left.\mathrm{LP}_{11 \mathrm{~b}}\right)$ are independently controlled through VOAs before spatial multiplexing performed by a photonic lantern (PL). At the receiver, the signals are demultiplexed by a second PL, digitized and processed. MDL/MDG estimation is performed by DSP based on the MIMO equalizer transfer function.

the $\mathrm{LP}_{11 \mathrm{~b}}$ mode is gradually attenuated, while the attenuation of the $\mathrm{LP}_{01}$ mode decreases in such a way that the total launch power is constant. For case 2, the $\mathrm{LP}_{01}$ mode is attenuated instead of the $\mathrm{LP}_{11 \mathrm{~b}}$ mode. In cases 3 and 4 , the $\mathrm{LP}_{11 \mathrm{a}}$ and $\mathrm{LP}_{11 \mathrm{~b}}$ modes are simultaneously attenuated, while the attenuation over the $\mathrm{LP}_{01}$ mode decreases.

TABLE II: VOA attenuation for MDL/MDG emulation

\begin{tabular}{cccc}
\hline \multirow{2}{*}{ Case } & \multicolumn{3}{c}{ Attenuation sweep } \\
\cline { 2 - 4 } & LP $_{\mathbf{0 1}}$ & LP $_{\mathbf{1 1 a}}$ & LP $_{\mathbf{1 1 b}}$ \\
\hline $\mathbf{1}$ & Decreases & Constant at $5 \mathrm{~dB}$ & $5 \mathrm{~dB}$ to $17 \mathrm{~dB}$ \\
$\mathbf{2}$ & 5 dB to $17 \mathrm{~dB}$ & Constant at $5 \mathrm{~dB}$ & Decreases \\
$\mathbf{3}$ & Decreases & $5 \mathrm{~dB}$ to $17 \mathrm{~dB}$ & $6 \mathrm{~dB}$ to $18 \mathrm{~dB}$ \\
$\mathbf{4}$ & Decreases & $5 \mathrm{~dB}$ to $17 \mathrm{~dB}$ & $5 \mathrm{~dB}$ to $17 \mathrm{~dB}$ \\
\hline
\end{tabular}

The induced MDL/MDG is estimated after DSP from the MIMO transfer function and averaged over 5 iterations. Fig. 4 shows $\mathrm{MDL} / \mathrm{MDG}_{\mathrm{pk}-\mathrm{pk}}$ and $\sigma_{\mathrm{mdg}}$ as a function of the ratio between the maximum and minimum attenuation for the four cases of Table II. From Fig. 4, the MDL/MDG emulation is effectively achieved by means of the different attenuation scenarios described in Table II. For the four attenuation scenarios, in general, the higher the ratio between attenuations, the higher the induced MDL/MDG. In case 2, at low attenuation ratios, the induced MDL/MDG decreases slightly before turning into an increasing curve. Such behavior comes from the fact that, in this configuration, the strongest mode, $\mathrm{LP}_{01}$, is more attenuated than modes $\mathrm{LP}_{11 \mathrm{a}}$ and $\mathrm{LP}_{11 \mathrm{~b}}$. Then, at some attenuation settings, the induced MDL/MDG counteracts the inherent system MDL/MDG. Using cases 3 and $4, \mathrm{MDL} / \mathrm{MDG}_{\mathrm{pk}-\mathrm{pk}}$ and $\sigma_{\mathrm{mdg}}$ achieve values higher than $15 \mathrm{~dB}$ and $6 \mathrm{~dB}$, respectively, as a consequence of the simultaneous attenuation of both $\mathrm{LP}_{11 \mathrm{a}}$ and $\mathrm{LP}_{11 \mathrm{~b}}$ modes. From these results, MDL/MDG emulation can be carried out by the independent attenuation of the single-mode signals before spatial multiplexing. Next, we sweep the attenuations from 0 $\mathrm{dB}$ to $17 \mathrm{~dB}$ in the 3 VOAs in order to generate a wide range of MDL/MDG. Fig. 5 shows the contour plot of MDL/MDG as a function of the launch powers in $\mathrm{LP}_{01}$ and $\mathrm{LP}_{11}$ modes. From the contour plot, MDL/MDG $\mathrm{Mk}_{\mathrm{pk}}$ and $\sigma_{\mathrm{mdg}}$ increase from the middle of the grid towards the top left corner and the bottom right corner, where the difference between the

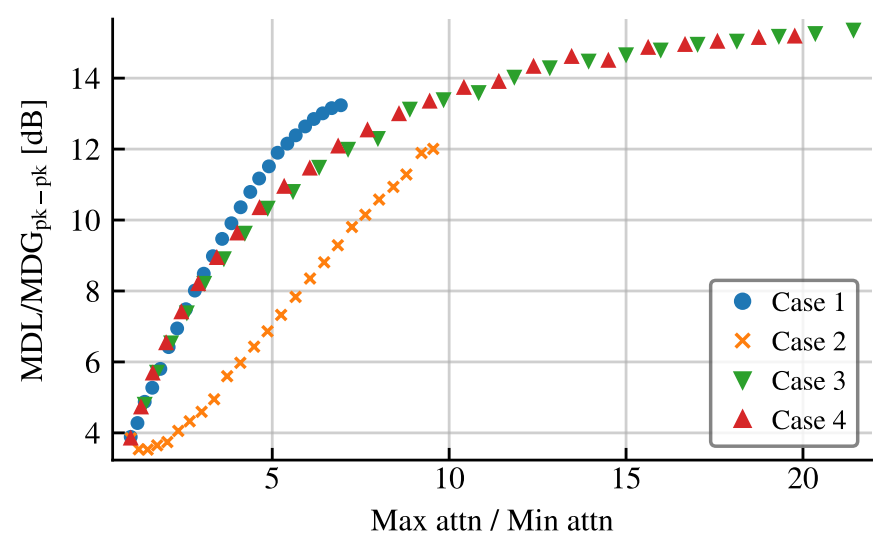

(a)

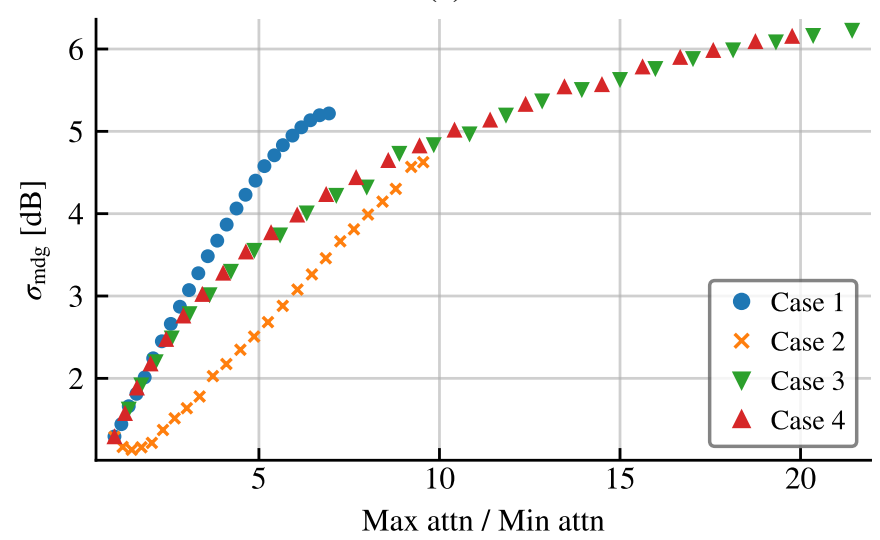

(b)

Fig. 4: MDL/MDG versus attenuation ratio at different VOA configurations. (a) Peak to peak MDL/MDG. (b) $\sigma_{\mathrm{mdg}}$.

launch powers is maximized. On the other hand, the region encompassing the diagonal between the bottom left corner and the top right corner presents low $\mathrm{MDL} / \mathrm{MDG}_{\mathrm{pk}-\mathrm{pk}}$ and $\sigma_{\mathrm{mdg}}$ as a consequence of the high similarity between the launch powers.

\section{CONCLUSION}

Space Division Multiplexing (SDM) is the only technology capable of supporting the sustained increase of traffic in core optical networks. In SDM with coupled channels, the MDL 


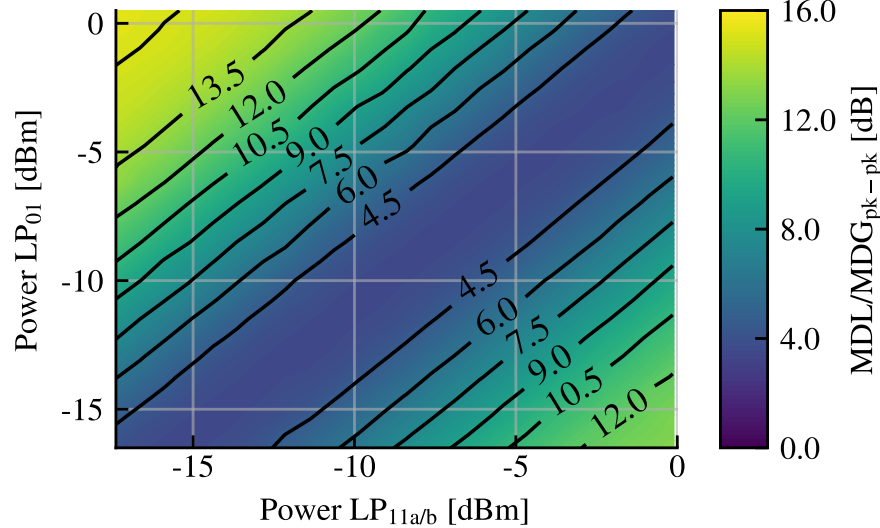

(a)

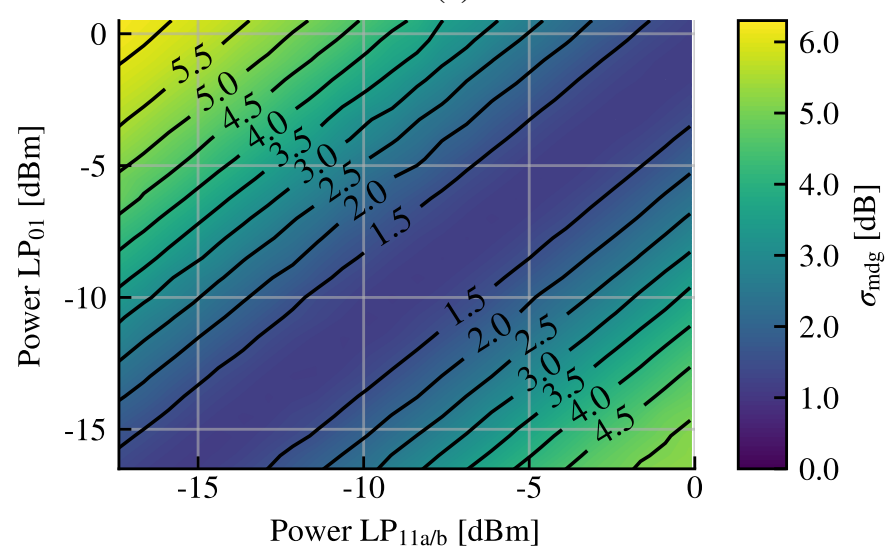

(b)

Fig. 5: Estimated MDL/MDG as a function of the launch powers in the $\mathrm{LP}_{01}$ and $\mathrm{LP}_{11}$ modes. (a) $\mathrm{MDL} / \mathrm{MDG}_{\mathrm{pk}-\mathrm{pk}}$ metric. (b) $\sigma_{\mathrm{mdg}}$ metric.

generated in optical components and the MDG generated in optical amplifiers fundamentally limit the system capacity. In small-scale laboratory experiments, the emulation of MDL/MDG is useful to investigate the MDL/MDG impact on the system performance and validate DSP algorithms in different propagation conditions. In this paper, we evaluate an MDL/MDG emulator based on variable optical attenuators and photonics lanterns. We assess the MDL/MDG emulation at different VOA attenuation configurations in a short-reach 3-mode SDM transmission system. We demonstrate the capability of the emulator to generate a wide range of MDL/MDG in laboratory setups. As future work, we will evaluate the performance of an alternative MDL/MDG emulator based on variable optical attenuators and photonics lanterns set up in the middle of the fiber span.

\section{ACKNOWLEDGMENTS}

This work was partially supported by FAPESP under grants 2018/25414-6, 2018/14026-5, 2017/25537-8, 2015/24341-7, 2015/24517-8 and by the Dutch TU/e-KPN flagship Smart Two project.

\section{REFERENCES}

[1] D. J. Richardson. "New optical fibres for high-capacity optical communications." Philosophical Transactions of the Royal Society A: Mathematical, Physical and Engineering Sciences 374 (2016): p.20140441.

[2] Peter J. Winzer, and Gerard J. Foschini. "Optical MIMO-SDM system capacities." Optical Fiber Communication Conference. IEEE, 2014.

[3] Alaelson C. Jatoba-Neto, et al. "Scaling SDM optical networks using full-spectrum spatial switching." IEEE/OSA Journal of Optical Communications and Networking 10.12 (2018): 991-1004.

[4] Omar D. Domingues, et al. "Achievable rates of space-division multiplexed submarine links subject to nonlinearities and power feed constraints." Journal of Lightwave Technology 35.18 (2017): 4004-4010.

[5] Roland Ryf, et al. "Coupled-core transmission over 7-core fiber." Optical Fiber Communication Conference. Optical Society of America, 2019.

[6] John van Weerdenburg, et al. "138-Tb/s mode-and wavelengthmultiplexed transmission over six-mode graded-index fiber." Journal of Lightwave Technology 36.6 (2018): 1369-1374.

[7] Roy G. H. Van Uden, et al. "Ultra-high-density spatial division multiplexing with a few-mode multicore fibre." Nature Photonics 8.11 (2014): 865.

[8] Roland Ryf, et al. "MIMO-Based Crosstalk Suppression in Spatially Multiplexed $3 \times 56-\mathrm{Gb} / \mathrm{s}$ PDM-QPSK Signals for Strongly Coupled Three-Core Fiber." IEEE Photonics Technology Letters 23.20 (2011): 1469-1471.

[9] Sakamoto, T., et al. "Randomly-coupled single-mode 12-core fiber with highest core density." 2017 Optical Fiber Communications Conference and Exhibition (OFC). IEEE, 2017.

[10] Keang-Po Ho, and Joseph M. Kahn. "Mode-dependent loss and gain: statistics and effect on mode-division multiplexing." Optics Express 19.17 (2011): 16612-16635.

[11] Peter J. Winzer, and Gerard J. Foschini. "MIMO capacities and outage probabilities in spatially multiplexed optical transport systems." Optics Express 19.17 (2011): 16680-16696.

[12] Darli A. A. Mello, et al. "Impact of polarization- and mode-dependent gain on the capacity of ultra-longhaul systems," Journal of Lightwave Technology, vol. 38 , no. 2 , pp. 303-318, 2020.

[13] Adriana Lobato, et al. "Mode-dependent-loss mitigation for modedivision multiplexed systems." Signal Processing in Photonic Communications. Optical Society of America, 2013.

[14] Adriana Lobato, et al. "On the mode-dependent loss compensation for mode-division multiplexed systems." 2013 15th International Conference on Transparent Optical Networks (ICTON). IEEE, 2013.

[15] Takayuki Mizuno, et al. "Mode dependent loss equaliser and impact of MDL on PDM-16QAM few-mode fibre transmission." 2015 European Conference on Optical Communication (ECOC). IEEE, 2015.

[16] Filipe Ferreira, et al. "Semi-analytical model for linear modal coupling in few-mode fiber transmission." 2012 14th International Conference on Transparent Optical Networks (ICTON). IEEE, 2012.

[17] Karthik Choutagunta, et al. "Characterizing mode-dependent loss and gain in multimode components." Journal of Lightwave Technology 36.18 (2018): 3815-3823

[18] Ruby S. B. Ospina, Darli A.A. Mello, and Chigo Okonkwo. "DSP-based Mode-dependent Loss and Gain Estimation in Coupled SDM Transmission." Optical Fiber Communication Conference. Optical Society of America, 2020.

[19] Tetsuya Hayashi, et al. "Record-low spatial mode dispersion and ultralow loss coupled multi-core fiber for ultra-long-haul transmission." Journal of Lightwave Technology 35.3 (2017): 450-457.

[20] Amado M. Velázquez-Benítez, et al. "Scaling photonic lanterns for space-division multiplexing." Scientific Reports 8.1 (2018): 1-9.

[21] Timothy A. Birks, et al. "The photonic lantern." Advances in Optics and Photonics 7.2 (2015): 107-167.

[22] Keang-Po Ho, et al. "Mode coupling and its impact on spatially multiplexed systems." Optical Fiber Telecommunications VI 17 (2013): 1386-1392

[23] Edson Porto da Silva, and Darko Zibar. "Widely linear equalization for IQ imbalance and skew compensation in optical coherent receivers." Journal of Lightwave Technology 34.15 (2016): 3577-3586. 